\title{
Dose perturbation study in a multichannel breast brachytherapy device
}

\author{
Eric D. Slessinger, MS', Rodney Fletcher, MS², Indra J. Das, PhD' \\ IIU Simon Cancer Center, Indiana University School of Medicine, Indianapolis, Indiana, 2Purdue University, West Lafayette, Indiana, USA
}

\begin{abstract}
Purpose: A study was conducted to determine the dosimetric effects resulting from air pockets and high atomic number $(Z)$ contrast medium within a multichannel breast brachytherapy device.

Material and methods: A 5-6 cm diameter Contura (SenoRx) brachytherapy device was inflated using $37 \mathrm{~cm}^{3}$ of saline. Baseline dose falloff from an HDR Iridium-192 source was measured with the Iridium source centered in the central channel and an anterior off-center channel. Data were collected at distances from 1 to $50 \mathrm{~mm}$. Comparison studies were conducted with identically inflated volume containing varied air pocket volumes $\left(1-4 \mathrm{~cm}^{3}\right)$ and concentrations of contrast solution (3\%, $6 \%$, and $9 \%$ by volume). Dose perturbation factors (DPF) were computed and evaluated.

Results: Dose perturbations due to air pockets and contrast solutions were observed. As the volume of air increased, the DPF increased by approximately $2.25 \% / \mathrm{cm}^{3}$. The effect was consistent for both channels. The contrast effects were more complex. The $3 \%$ contrast media had minimal dose perturbation. The $6 \%$ contrast solution caused dose reduction of $1.0 \%$ from the central channel but $1.5 \%$ dose increase from the anterior channel. The $9 \%$ contrast solution caused dose reductions by $4.0 \%$ (from central channel) and 3.0\% (from anterior channel). The DPF from all contrast solutions moderated with increasing distance.
\end{abstract}

Conclusions: Dose perturbations due to air pockets and high-Z contrast solution can be significant. It is important to control these effects to avoid dose errors.

Key words: brachytherapy, breast cancer, dose perturbation, PBI.

\section{Purpose}

The Contura Multi-lumen Balloon (MLB) by SenoRx is a five-channel breast brachytherapy balloon developed for accelerated partial breast irradiation (APBI) using high dose rate Iridium-192. The device is designed to improve upon the utility of single channel breast brachytherapy by offering multiple channels for the sake of dose optimization. Because of its close proximity, the single channel device is not always suitable, as it can lead to unacceptably high skin dose, resulting in poor cosmesis [1, 2]. The Contura MLB design includes four stable treatment channels that surround a fifth central channel. This geometry offers greater ability to optimize the treatment dose distribution, especially with regard to skin, rib and lung. Target tissue conformance to the balloon surface is another important consideration in this type of APBI. Ideally, no gaps between balloon and target tissue should occur. However, up to $10 \%$ of the planning target volume (PTV) may be displaced by air or fluid and still be considered acceptable [3]. The Contura has a vacuum port that can remove air and fluid from the surrounding lumpectomy cavity (other devices do not have this feature) and thereby improve tissue conformance to the balloon surface [4].

The Contura device is inflated with a solution of saline and iodine contrast (Isovue-M 300, Bracco Diagnostics) to a volume ranging from $28-64.5 \mathrm{~cm}^{3}(4-5 \mathrm{~cm}$ diameter Contura), depending on lumpectomy cavity size. The vendor recommends a $3 \%$ contrast solution by volume with the saline to assure optimal imaging using either CT or simulator. The introduction of the solution may also allow an air bubble to enter. Most commercial treatment planning systems are not capable of computing the perturbation effects that may result from air pockets or contrast material [5]. Earlier studies have investigated dose perturbation effects associated with certain partial breast brachytherapy devices [6-12]. This study evaluates the dosimetric effects of high atomic number contrast and air pockets for the Contura device.

\section{Material and methods}

The experimental setup for the dose perturbation measurements used a water phantom with a waterproof Markus parallel plate ionization chamber (PTW) and K602 electrometer (CNMC Best). The chamber was supported on a motorized vertical drive assembly with the incident surface overlying the upper Contura surface. The Contura was inflated to $37 \mathrm{~cm}^{3}$ (42 $\mathrm{mm}$ diameter) and supported horizontally in a ring stand with a clamp. The upper Contura 

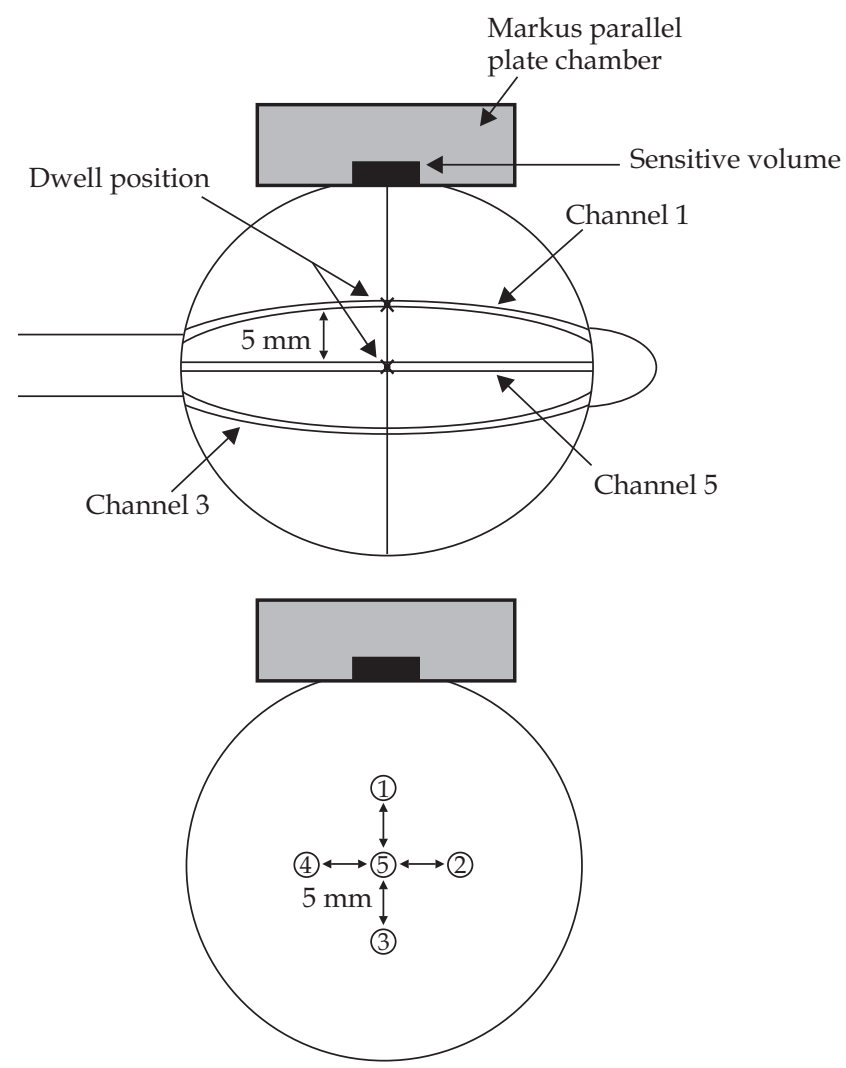

surface was positioned $8 \mathrm{~cm}$ below the water surface. Two flexible needle source transfer tubes connected the Nucletron Microselectron indexer to the central Contura [SenoRx] channel (\#5) and to the channel oriented closest to the ionization chamber (channel \#1). The channel attachments were made above the water surface by bending the Contura stem vertically. Figure 1 shows the measurement setup and geometry.

The determination of the "sweetspot" (Ir-192 source position that resulted in the maximum ionization current) was performed for each channel and repeated for each experimental condition. Baseline ionization measurements were made at distances from 1-51 mm from the Contura surface without any air bubble or contrast. The following seven test conditions were achieved using a $60 \mathrm{cc}$ syringe and then tested for comparison to determine dose perturbation effects: $1 \mathrm{~cm}^{3}, 2 \mathrm{~cm}^{3}, 3 \mathrm{~cm}^{3}$ and $4 \mathrm{~cm}^{3}$ air bubble, and $3 \%, 6 \%$ and $9 \%$ contrast solutions. Corrections were applied to account for temperature and pressure variations and for the source decay during several days of the measurements. The experimental setup was performed twice and the results reported are the average of the two data sets. DPF was defined as the ratio of dose with and without air or contrast medium.

\section{Results}

Figures 2 and 3 demonstrate that higher doses by $2-4 \%$ result from the introduction of $1-3 \mathrm{~cm}^{3}$ of air. The $4 \mathrm{~cm}^{3}$ air pocket significantly increases the perturbation effect to $7 \%$. The dimensions of the air volumes were not evaluated but these results indicate that the path length of air toward

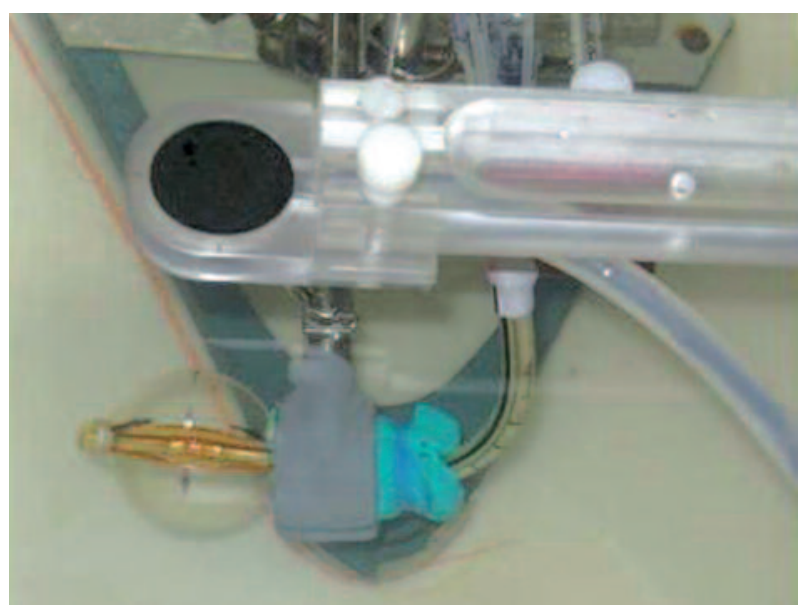

Fig. 1. The incident surface of the parallel plate chamber was positioned adjacent to the upper surface of the Contura (SenoRx) and a motorized support raised the chamber for data collection (right). The outer Contura channels are $5 \mathrm{~mm}$ from the central channel at mid cross-section (left)

the point of measurement is not proportional to the volume of air introduced. With two sets of data taken, the uncertainty in the measurements was in the range of $0.9 \pm 2.4 \%$. Figures 4 and 5 demonstrate that the $3 \%$ contrast media solution causes minimal effect. The $6 \%$ contrast solution reduces dose by $1-2 \%$. The $9 \%$ solution results in $4 \%$ lower dose close to the Contura balloon and moderates with distance to $2-3 \%$. The air pocket and the contrast experiments indicate that the perturbation effects diminish with increasing distance. The central and off-center channel results for each measurement condition are similar but more pronounced by about $1 \%$ for the central channel. For the two measurement sessions the reproducibility of air volume perturbation results did not vary by more than $\pm 0.9 \%$ with standard deviation of $\pm 0.3 \%$. The reproducibility of the contrast perturbation experiments was within $\pm 2.4 \%$ for the central channel and $\pm 1.5 \%$ for the off-center channel with standard deviations within $\pm 0.3 \%$.

\section{Discussion}

Dose perturbations resulting from air pockets and high atomic number contrast have been studied in accelerated partial brachytherapy devices. Based on earlier dose perturbation studies $[7-9,11,12]$ of the single channel Mammosite, similar results would be expected for the Contura, at least from the central channel 5. Those studies demonstrated that high $\mathrm{Z}$ contrast causes dose reduction while the presence of air leads to increased dose. The measured data of this study, shown in Figs. 2-5, support those findings. The perturbation is not limited to a local effect. Some 


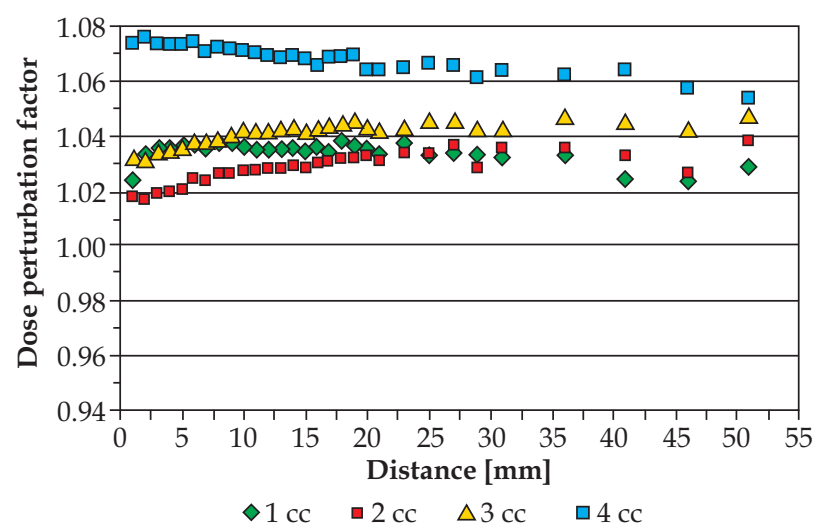

Fig. 2. 1-4 cc Air Bubble, No Contrast, Channel 5. Dose perturbations due to air pockets with the HDR Iridium source in the central Contura (SenoRx) channel (\#5) (Data collected $1 \mathrm{~mm}$ to $51 \mathrm{~mm}$ from Contura surface)

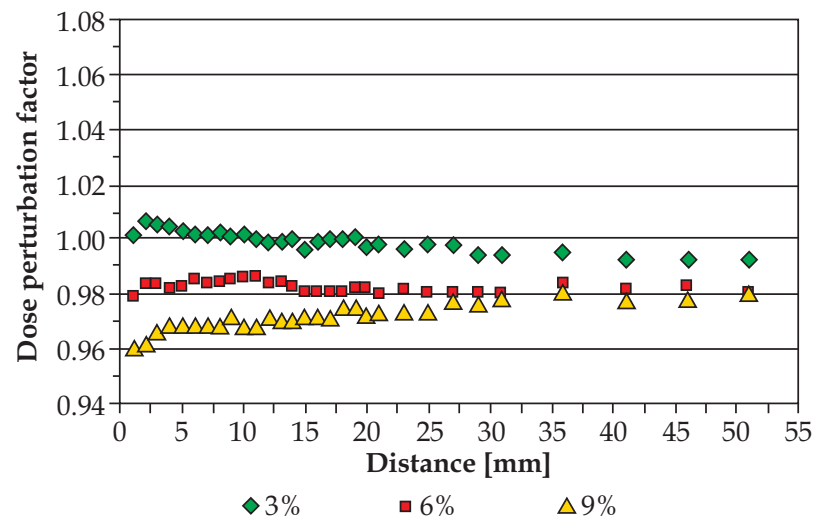

Fig. 4. 3-9\% Contrast, No Air Bubble, Channel 5. Dose perturbations due to different Iodinated contrast solutions (by volume) with the HDR Iridium source in the central Contura (SenoRx) channel (\#5) (Data collected $1 \mathrm{~mm}$ to $51 \mathrm{~mm}$ from Contura surface)

moderation does occur with increasing distance, but the perturbation effects do persist. This study also demonstrates that channel location does not impact significantly on the perturbation effects. Although air bubble dimensions were not evaluated, the experimental conditions would be comparable in the clinical situation.

Measurement uncertainty must be considered when comparing the results of this study to others. Brachytherapy dose measurement precision is always limited in non rigid phantoms. In this study deflection and compression of the Contura balloon contribute to the measurement uncertainty that ranged from \pm 0.9 to $\pm 2.4 \%$. This represents a limitation on the conclusions that can be drawn as well as differences from results previously reported for the Mammosite. In this study the effect of air appears to be more significant while the effect of contrast is less. Differences in applicator design may also have some related effects. However, the trends are clear and consistent and demonstrate the importance of following vendor instructions and

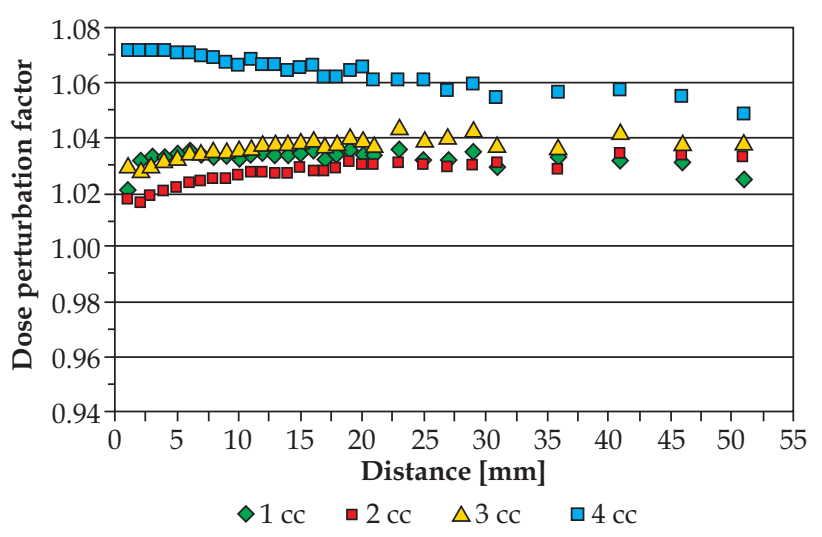

Fig. 3. 1-4 cc Air Bubble, No Contrast, Channel 1. Dose perturbations due to air pockets with the HDR Iridium source in the Contura (SenoRx) channel \#1 (Data collected $1 \mathrm{~mm}$ to $51 \mathrm{~mm}$ from Contura surface)

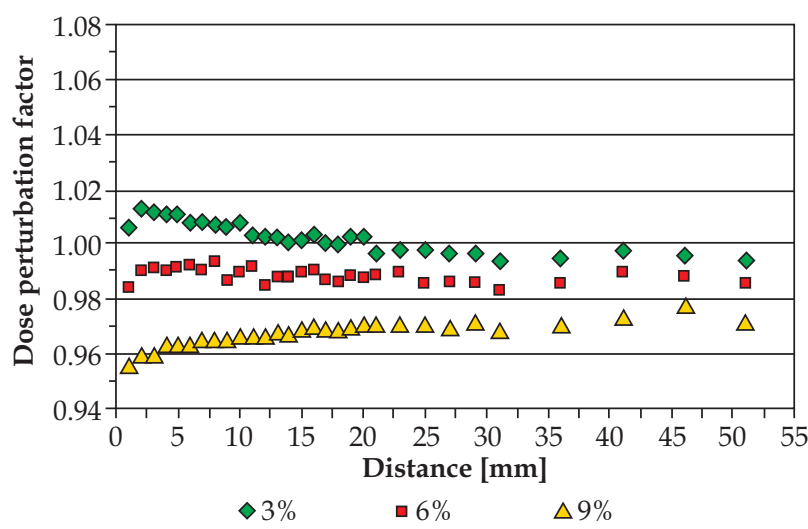

Fig. 5. 3-9\% Contrast, No Air Bubble, Channel 1. Dose perturbations due to different Iodinated contrast solutions (by volume) with the HDR Iridium source in the Contura (SenoRx) channel \#1 (Data collected $1 \mathrm{~mm}$ to $51 \mathrm{~mm}$ from Contura surface)

sound procedures to ensure accurate dose delivery. Vendor instructions recommend 3\% contrast solution by volume and that is shown here to cause clinically insignificant dose perturbation effect.

\section{Conclusions}

Dose perturbation effects due to air and high $\mathrm{Z}$ contrast in a multichannel breast brachytherapy device have been measured. The results reported here are similar to earlier reported results for a single central channel device. In the absence of inhomogeneity correction, commercial brachytherapy planning software cannot predict the results measured in these studies. Incorporation of CT data for planning should be developed to offer improved dose computation accuracy. For that reason, it is important to follow the appropriate guidelines for the use of contrast and to minimize the presence of air in order to limit dose perturbation effects in patient treatment. 


\section{References}

1. Edmundson GK, Vicini FA, Chen PY et al. Dosimetric characteristics of the MammoSite RTS, a new breast brachytherapy applicator. Int J Radiat Oncol Biol Phys 2002; 52: 1132-1139.

2. Keisch M, Vicini F, Kuske RR et al. Initial clinical experience with the MammoSite breast brachytherapy applicator in women with early-stage breast cancer treated with breast-conserving therapy. Int J Radiat Oncol Biol Phys 2003; 55: 289-293.

3. NSABP B-39/RTOG 0413 Protocol. A randomized phase III study of conventional whole breast irradiation (WBI) versus partial breast irradiation (PBI) for women with stage 0,1 or 2 breast cancer; available at: http://www.rtog.org/members/protocols/0413/0413.pdf.

4. Arthur DW, Vicini FA, Todor DA et al. Improvements in critical dosimetric endpoints using the Contura multilumen balloon breast brachytherapy catheter to deliver accelerated partial breast irradiation: preliminary dosimetric findings of a phase iv trial. Int J Radiat Oncol Biol Phys 2011; 79: 26-33.

5. Rivard MJ, Venselaar JLM, Beaulieu L. The evolution of brachytherapy treatment planning. Med Phys 2009; 36: 21362153.

6. Bensaleh S, Bezak E. The impact of uncertainties associated with MammoSite brachytherapy on the dose distribution in the breast. J Appl Clin Med Phys 2011; 12: 82-96.

7. Cheng CW, Mitra R, Li XA et al. Dose perturbations due to contrast medium and air in mammosite treatment: an experimental and Monte Carlo study. Med Phys 2005; 32: 2279-2287.

8. Huang YJ, Blough M. Dosimetric effects of air pocket sizes in MammoSite treatment as accelerated partial breast irradiation for early breast cancer. J Appl Clin Med Phys 2010; 11: 2932.

9. Kassas B, Mourtada F, Horton JL et al. Contrast effects on dosimetry of a partial breast irradiation system. Med Phys 2004; 31: 1976-1979.

10. Richardson SL, Pino R. Dosimetric effects of an air cavity for the SAVI partial breast irradiation applicator. Med Phys 2010; 37: 3919-3926.

11. Ye S-J, Brezovich IA, Shen S et al. Dose errors due to inhomogeneities in balloon catheter brachytherapy for breast cancer. Int J Radiat Oncol Biol Phys 2004; 60: 672-677.

12. Zhang Z, Parsai EI, Feldmeier JJ. Three-dimensional quantitative dose reduction analysis in MammoSite balloon by Monte Carlo calculations. J Appl Clin Med Phys 2007; 8: 2669. 